\title{
Carbon nanoparticles as a multimodal thermoacoustic and photoacoustic contrast agent
}

Xin Cai, Lina Wu, Wenxin Xing, Jun Xia, Liming Nie, et al.

Xin Cai, Lina Wu, Wenxin Xing, Jun Xia, Liming Nie, Ruiying Zhang, Gregory M. Lanza, Baozhong Shen, Dipanjan Pan, Lihong V. Wang, "Carbon nanoparticles as a multimodal thermoacoustic and photoacoustic contrast agent," Proc. SPIE 8581, Photons Plus Ultrasound: Imaging and Sensing 2013, 858140 (4 March 2013); doi: 10.1117/12.2005064

SPIE. Event: SPIE BiOS, 2013, San Francisco, California, United States 


\title{
Carbon Nanoparticles as a Multimodal Thermoacoustic and Photoacoustic Contrast Agent
}

\author{
Xin Cai, ${ }^{1}$ Lina Wu, ${ }^{2,3}$ Wenxin Xing, ${ }^{1}$ Jun Xia, ${ }^{1}$ Liming Nie, ${ }^{1}$ Ruiying Zhang, ${ }^{1}$ \\ Gregory M. Lanza, ${ }^{2}$ Baozhong Shen, ${ }^{3}$ Dipanjan Pan, ${ }^{2}$ and Lihong V. Wang ${ }^{1, *}$ \\ ${ }^{1}$ Optical Imaging Laboratory, Department of Biomedical Engineering, Washington University in St. \\ Louis, St. Louis, Missouri 63130 \\ ${ }^{2}$ Division of Cardiology, Washington University School of Medicine, St. Louis, MO 63108 \\ ${ }^{3}$ Molecular Imaging Center and Radiology Department, $4{ }^{\text {th }}$ Affiliated Hospital of Harbin Medical \\ University, Harbin, China
}

\begin{abstract}
We demonstrated the potential of carbon nanoparticles (CNPs) as exogenous contrast agents for both thermoacoustic (TA) tomography (TAT) and photoacoustic (PA) tomography (PAT). In comparison to deionized water, the CNPs provided a four times stronger signal in TAT at $3 \mathrm{GHz}$. In comparison to blood, The CNPs provided a much stronger signal in PAT over a broad wavelength range of $450-850 \mathrm{~nm}$. Specifically, the maximum signal enhancement in PAT was 9.4 times stronger in the near-infrared window of $635-670 \mathrm{~nm}$. In vivo blood-vessel PA imaging was performed non-invasively on a mouse femoral area. The images, captured after the tail vein injection of CNPs, show a gradual enhancement of the optical absorption in the vessels by up to $230 \%$. The results indicate that CNPs can be potentially used as contrast agents for TAT and PAT to monitor the intravascular or extravascular pathways in clinical applications.
\end{abstract}

Keywords: Contrast agents; carbon; photoacoustic tomography; thermoacoutic tomography; nanoparticle.

\section{INTRODUCTION}

Thermoacoustic (TA) tomography (TAT) and photoacoustic (PA) tomography (PAT) are non-invasive techniques that uniquely synergize pure ultrasound and pure radio frequency (rf) and optical imaging, allowing both satisfactory spatial resolution and high soft-tissue contrast. ${ }^{1-2}$ The technique is based on the detection of acoustic waves from an object that absorbs pulsed or intensity-modulated electromagnetic energy ( $\mathrm{rf}$ band in TAT and laser in PAT). The absorption can be associated with endogenous molecules, such as water/ion, hemoglobin, and melanin. For instance, due to the high concentration of hemoglobin (12 to $15 \mathrm{~g} / \mathrm{dl}$ ), blood inherently has a strong optical absorption which allows the visualization of blood vessels. However, the absorption of nonvascular tissues (e.g., lymph nodes) or intravascular biosignatures (e.g., integrins) is insufficient. Thus, exogenous contrast agents such as optical dyes, gold nanoparticles, copper nanoparticles, and carbon nanotubes are needed for TAT/PAT in these cases. ${ }^{3-6}$ TAT and PAT have been developed for different applications in rodent models, such as breast cancer imaging, brain structural and functional imaging, blood oxygenation and flow velocity monitoring, and tumor angiogenesis. ${ }^{1,7-8}$

* Corresponding author: $\underline{\text { hwang@ } a \text { biomed.wustl.edu }}$

Photons Plus Ultrasound: Imaging and Sensing 2013, edited by Alexander A. Oraevsky, Lihong V. Wang, Proc. of SPIE Vol. 8581, 858140 - (C) 2013 SPIE · CCC code: 1605-7422/13/\$18 - doi: 10.1117/12.2005064 
Although a lot of contrast agents are available for TAT and PAT, they have their disadvantages. For example, optical dyes easily cause skin staining; gold nanoparticles are high in cost and have complicated chemistries; and the toxicity of copper nanoparticles and carbon nanotubes is arguable. Therefore, new contrast agents still deserve exploration. We reveal a simple and commercially amenable synthetic methodology for creating optically active carbon nanoparticles (CNP). CNPs are derived from commercial food-grade honey. Compare to the previously explored particles (gold, copper, carbon nanotube etc.), CNPs are significantly smaller ( $\sim \mathrm{nm}$ in diameter), enabling rapid clearance properties.

In this study, we explore the rf and optical absorbtion properties of carbon nanoparticles (CNPs) as multimodal contrast agents for both TAT and PAT. Then, we further monitor the pharmacokinetics of CNPs in blood vessels in mice in vivo using PAT. The results could be greatly beneficial for monitor the intravascular or extravascular pathways using TAT/PAT together with other structural imaging modalities.

\section{MATERIALS AND METHODS}

\subsection{Synthesis of optically active carbon nanoparticles}

Commercial grade honey (Great Value ${ }^{\mathrm{TM}}$ Clover Honey $1 \mathrm{wt} \%$; batch composition- fructose: $38 \%$, glucose: $31 \%$, maltose: $7.1 \%$, sucrose: $1.3 \%$, higher sugars: $1.5 \%$, water: $17.2 \%$ ) is suspended with an organic macromolecular passivating agent (8wt\%; PEG400), purged with argon, and heated in a domestic microwave oven for $30 \mathrm{~min}$. Microwave power was set at $1200 \mathrm{~W}$ with an output power of $50 \%$. The product was then purified by repeated centrifugation in water.

\subsection{Thermoacoustic system}

For thermoacoustic excitation, a 3.0-GHz microwave generator with pulse width of $0.6 \mu$ s and repetition rate of $10 \mathrm{~Hz}$ was employed. The pulses were guided toward the target through a horn antenna. The size of the antenna opening was 11 $\mathrm{cm} \times 7 \mathrm{~cm}$. The fluence is $0.45 \mathrm{~mJ} / \mathrm{cm}^{2}$ at the opening of the antenna, which is within the safety standard. The test samples were placed in a plastic tank filled with mineral oil for ultrasonic coupling. A 1-MHz spherically focused transducer with a bandwidth of $70 \%$ (V314, Panametrics, Olympus) was used to receive TA signals.

\subsection{Photoacoustic system}

The schematic of the system has been reported before. ${ }^{9}$ For photoacoustic excitation, three different light sources were employed for different spectra range or imaging speed: 1) a tunable OPO laser (450-685 nm, Vibrant (HE) 355 I, OPOTEK), pulse width $5 \mathrm{~ns}$, pulse repetition rate $10 \mathrm{~Hz} ; 2)$ a tunable Ti:sapphire laser (730-850 nm, LT-2211A, LOTIS TII) pumped by a Q-switched Nd:YAG (LS-2137/2, LOTIS TII), pulse width $<15 \mathrm{~ns}$, pulse repetition rate $10 \mathrm{~Hz}$; and 3) a dye laser (CBR-D, Sirah) pumped by a Nd:YLF laser (INNOSLAB, Edgewave), pulse width $<7 \mathrm{ns,} \mathrm{pulse} \mathrm{repetition}$ rate up to $5 \mathrm{kHz}$. The first two sources were used for the measurement of the PA spectrum of CNPs. The third source was used for fast PA imaging in vivo. The fluence is $<1 \mathrm{~mJ} / \mathrm{cm}^{2}$ on the sample surface. For photoacoustic detection, a focused ultrasonic transducer with $50 \mathrm{MHz}$ central frequency (V214-BB-RM, Olympus NDT) was employed. The transducer surface was immersed in water for ultrasonic coupling. The optical and ultrasonic foci were configured coaxially and confocally. This system could achieve $45 \mu \mathrm{m}$ lateral resolution, $15 \mu \mathrm{m}$ axial resolution, and more than 3 mm penetration depth.

\subsection{Animals}

All animal experiments were performed in accordance with protocols approved by the Washington University Department of Comparative Medicine and the Animal Studies Committee. Athymic nude mice were obtained from Harlan and housed in the animal facility at Washington University. During the experiments, the animals were anesthetized by administration of gaseous isoflurane (2\%, Butler Inc., Dublin) and aseptically prepared. 

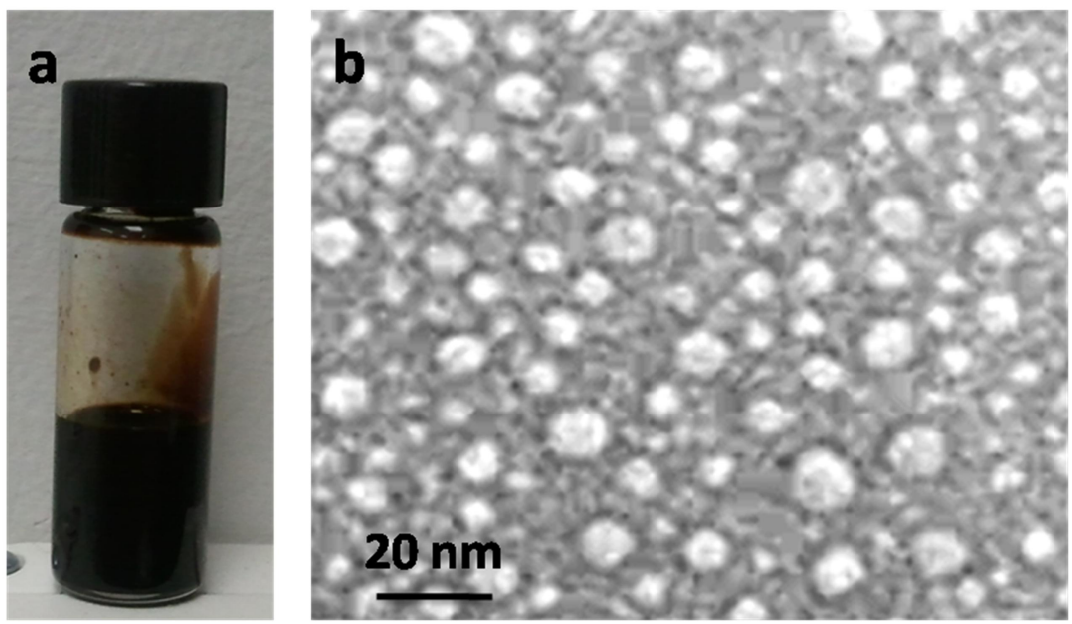

Fig. 1. (a) A typical photograph of solution of CNPs in water (33 vol\%). (b) A scanning electron microscopy image of CNPs in drydispersed state.

\section{RESULTS}

As-synthesized CNPs are soluble in water. Fig. 1a shows a typical photograph of solution of CNPs in water (33 vol\%). From the scanning electron microscopy (SEM) image (Fig. 1b), it is clear that CNPs are spherical and exhibit a diameter (number-averaged) of $8 \pm 2 \mathrm{~nm}$.

Water and ions are two well-known sources of microwave absorbers in human, and they generate strong TA signals. Therefore, to show that CNPs can function as a contrast agent for TA, we first compared the TA signal of CNPs with that of water. Fig. 2a shows the TA signals obtained from a low-density polyethylene (LDPE) vial (I.D. $=6 \mathrm{~mm}$ and $1.5 \mathrm{cc}$ volume) filled with CNPs (33 vol\%) or deionized (DI) water. The LDPE vial does not generate any measurable TA signal. ${ }^{10}$ At $3 \mathrm{GHz}$, CNPs (33 vol\%) exhibited more than four-fold TA signal enhancement compared with DI water (Fig. 2).

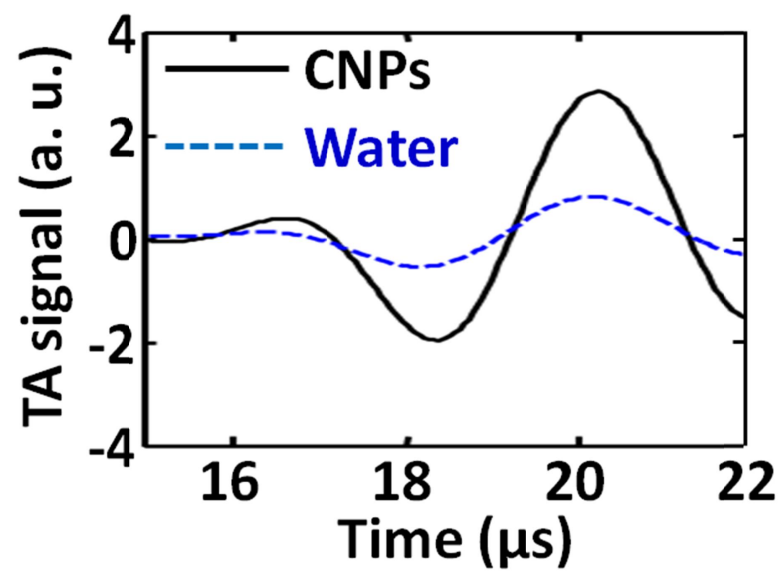

Fig. 2. TA signals at $3 \mathrm{GHz}$ from a LDPE vial (I.D. $=6 \mathrm{~mm}$ and $1.5 \mathrm{cc}$ volume) filled with CNPs (33 vol \%) and DI water 
Similarly, hemoglobin is a dominant optical absorber in humans and generates strong PA signals. We compared the PA efficacy of CNPs with blood at various excitation wavelengths. PA signals were obtained from tygon tubes filled with CNPs (33 vol \%) and rat whole blood. The tube is transparent, and therefore, it does not produce any measurable PA signal. Fig. 3a shows the ratio of the peak-to-peak PA signal amplitude of CNPs (33 vol \%) to that of blood. The excitation wavelength range was $450-850 \mathrm{~nm}$, excluding the range of $685-730 \mathrm{~nm}$ due to the gap between two lasers. The PA signal obtained from CNPs was much stronger than or comparable to blood over such broad wavelength range, which covered both visible and near infrared (NIR) light. For example, at the excitation $\lambda=670 \mathrm{~nm}, \mathrm{CNPs}(33$ vol \%) exhibited 9.4 times PA signal enhancement compared to blood (Fig. 3b).
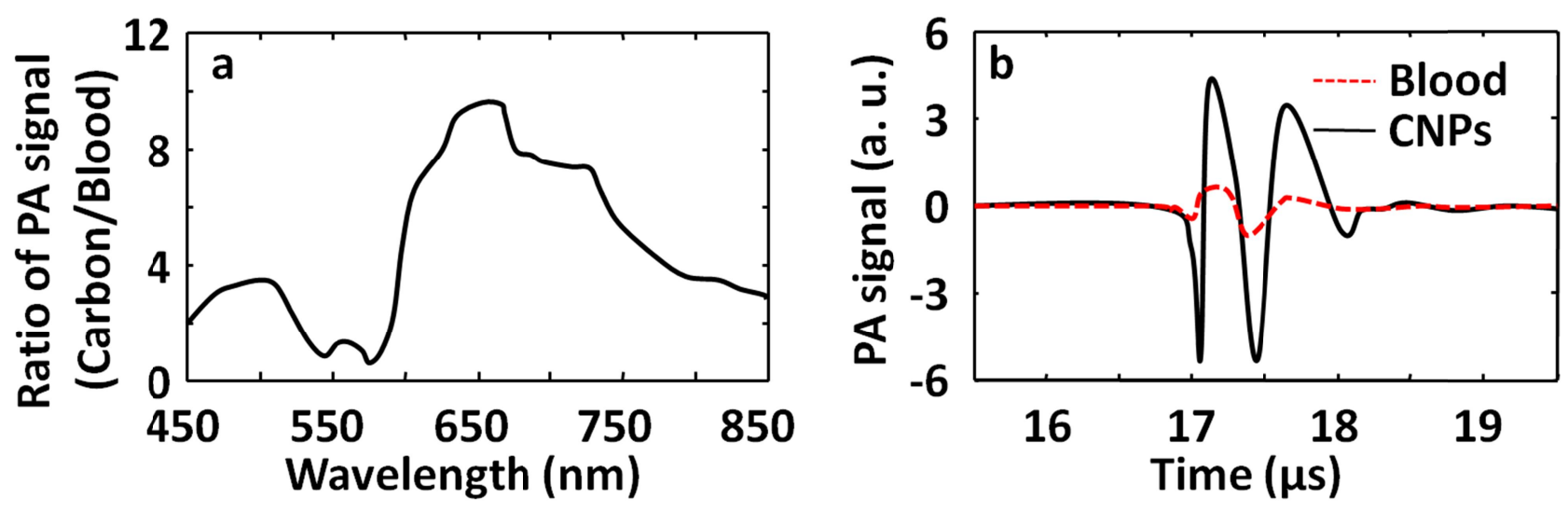

Fig. 3. (a) Ratio of the peak-to-peak PA signals generated from CNPs (33 vol \%) to those of blood at different wavelengths. (b) PA signals generated from CNPs and rat blood at the wavelength of $670 \mathrm{~nm}$.

Preliminary in vivo blood-vessel PA imaging was performed non-invasively on a mouse femoral area. The excitation wavelength was tuned to $650 \mathrm{~nm}$. Before CNP injection, a PA image of the femoral was acquired as the base-line (Fig. 4a). After the CNPs $(25 \mathrm{vol} \%, 100 \mu \mathrm{L})$ were intravenously injected through the tail vein, a series of PA images were taken at the same position with an interval of 1-2 min. At 6 min post-injection (Fig. 4b), the vasculature showed greater clarity than that in Fig. 4a did, because the exogenous contrast agent, CNPs, induced optical absorption increase The differential image in Fig. 4c is a result of the subtraction of Fig. 4a from Fig. 4b. This image indicates the distribution of differential optical absorption in the vascular caused by the exogenous contrast agent.
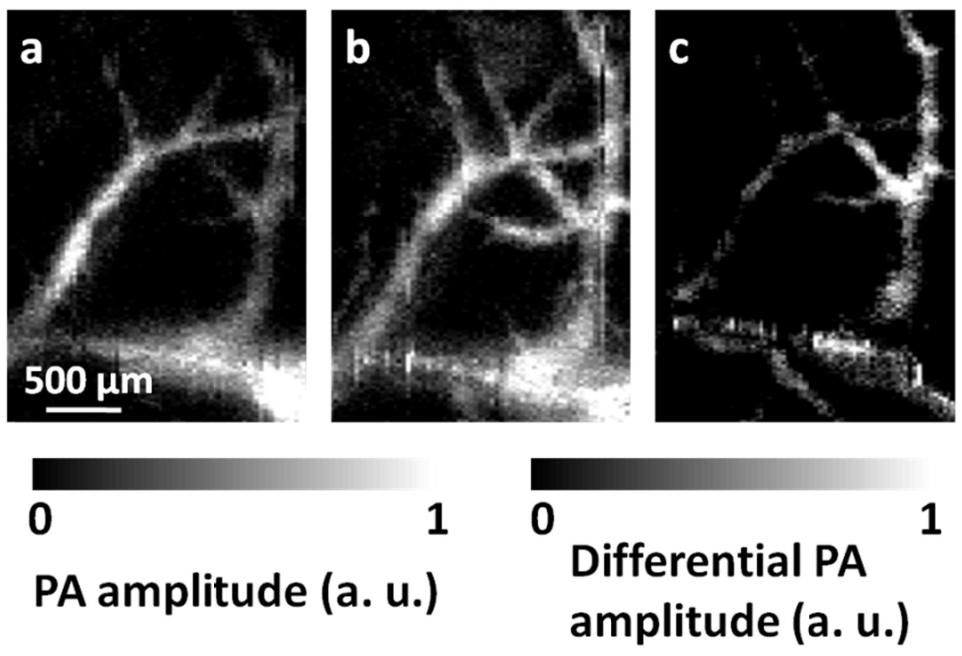

Fig. 4. Noninvasive PA imaging the femoral area of a mouse in vivo employing CNPs as contrast agents. (a) PA image acquired before the injection of CNPs. (b) PA image acquired at 6 min post-injection of CNPs $(25 \mathrm{vol} \%, 100 \mu \mathrm{L})$ through the tail vein. (c) Differential image that was obtained by subtracting the pre-injection image (a) from the post-injection image (b). 


\section{CONCLUSION}

In summary, we have successfully demonstrated the potential of carbon nanoparticles (CNPs) as exogenous contrast agents for both thermoacoustic tomography (TAT) and photoacoustic tomography (PAT). The CNPs provided more than 4 times signal enhancement in TAT at $3 \mathrm{GHz}$ and more than 9.4 times signal enhancement in PAT in the NIR window of 635-670 nm. The results indicate that CNPs can be potentially used as contras agents for TAT and PAT together with other structural imaging modalities to monitor the intravascular or extravascular pathways in clinical applications.

\section{ACKNOWLEDGMENTS}

Our work was sponsored by NIH grants R01 EB000712, R01 EB008085, R01 CA140220, R01 CA157277, R01 CA159959, U54 CA136398, and DP1 EB016986-NIH Director's Pioneer Award. L.V.W. has a financial interest in Microphotoacoustics, Inc. and Endra, Inc., which, however, did not support this work. Others claim no competing financial interests.

\section{REFERENCES}

[1] L. V. Wang and S. Hu, "Photoacoustic tomography: in vivo imaging from organelles to organs," Science 335, 14581462 (2012).

[2] L. V. Wang, "Ultrasound-mediated biophotonic imaging: a review of acousto-optical tomography and photo-acoustic tomography, " Dis Markers 19(3), 123-138 (2004).

[3] M.-L. Li, J. T. Oh, X. Y. Xie, G. Ku, W. Wang, C. Li, G. Lungu, G. Stoica, and L. V. Wang, "Simultaneous molecular and hypoxia imaging of brain tumors in vivo using spectroscopic photoacoustic tomography," Proc of the IEEE 96, 481-489 (2008).

[4] X. Cai, W. Li, C. Kim, Y. Yuan, L. V. Wang, and Y. Xia, "In vivo quantitative evaluation of the transport kinetics of gold nanocages in a lymphatic system by noninvasive photoacoustic tomography," ACS Nano 5(12), 9658-9667 (2011).

[5] D. Pan, X. Cai, C. Yalaz, A. Senpan, K. Omanakuttan, S. A. Wickline, L. V. Wang, and G. M. Lanza, "Photoacoustic sentinel lymph node imaging with self-assembled copper neodecanoate nanoparticles," ACS Nano 6(2), 1260-1267 (2012).

[6] X. Cai, B. S. Paratala, S. Hu, B. Sitharaman, and L. V. Wang, "Multiscale photoacoustic microscopy of single-walled carbon nanotube-incorporated tissue engineering scaffolds," Tissue Eng Part C: Methods 18(4), 310-317 (2012).

[7] C. Kim, C. Favazza, L. V. Wang, "In vivo photoacoustic tomography of chemicals: high-resolution functional and molecular optical imaging at new depths," Chem Rev 110, 2756-2782 (2010).

[8] S. Hu, L. V. Wang, "Photoacoustic imaging and characterization of the microvasculature," J Biomed Opt 15(1), 011101 (2010).

[9] H. F. Zhang, K. Maslov, G. Stoica, L. V. Wang, "Functional photoacoustic microscopy for high-resolution and noninvasive in vivo imaging," Nat Biotechnol 24, 848-851 (2006).

[10] M. Pramanik, M. Swierczewska, D. Green, B. Sitharaman, and L. V. Wang, "Single-walled carbon nanotubes as a multimodal — thermoacoustic and photoacoustic — contrast agent" J Biomed Opt 14 (3), 034018 (2009). 